\title{
Strong coupling of plasmon and nanocavity modes for dual band, near-perfect absorbers and ultrathin photovoltaics
}

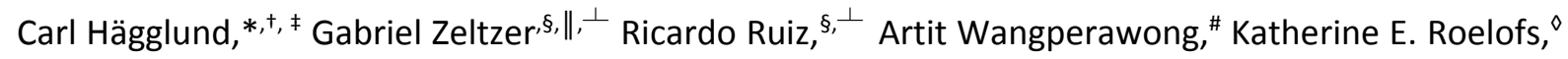
and Stacey F. Bent ${ }^{*,+}$

†Department of Chemical Engineering, Stanford University, Stanford, California 94305, USA $\S$ HGST, a Western Digital company, San Jose, California 95135, USA \#Department of Electrical Engineering, Stanford University, Stanford, California 94305, USA $\checkmark$ Department of Materials Science and Engineering, Stanford University, Stanford, California, 94305, USA.

'These authors contributed equally.

*carl.hagglund@angstrom.uu.se, sbent@stanford.edu

\section{Present Addresses:}

${ }^{\ddagger}$ Division of Solid State Electronics, Department of Engineering Sciences, Uppsala University, 75121 Uppsala, Sweden

"Waves Audio Ltd, Tel Aviv 6701101, Israel

\#Department of Electrical Engineering, Faculty of Engineering, King Mongkut's University of Technology Thonburi, Bangkok 10140, Thailand.

\section{Contents}

- Example fit to ellipsometry data

- Optical properties of gold nanodots and their effect on the sample absorptance

- Effect of aluminum interband transitions on overall absorptance

- Effect of coating filling factor on overall and individual layer absorptance

- Comparison of the absorption in SnS with and without gold nanodots

Figure S1-S5, 5 pages 


\section{Example fit to ellipsometry data}

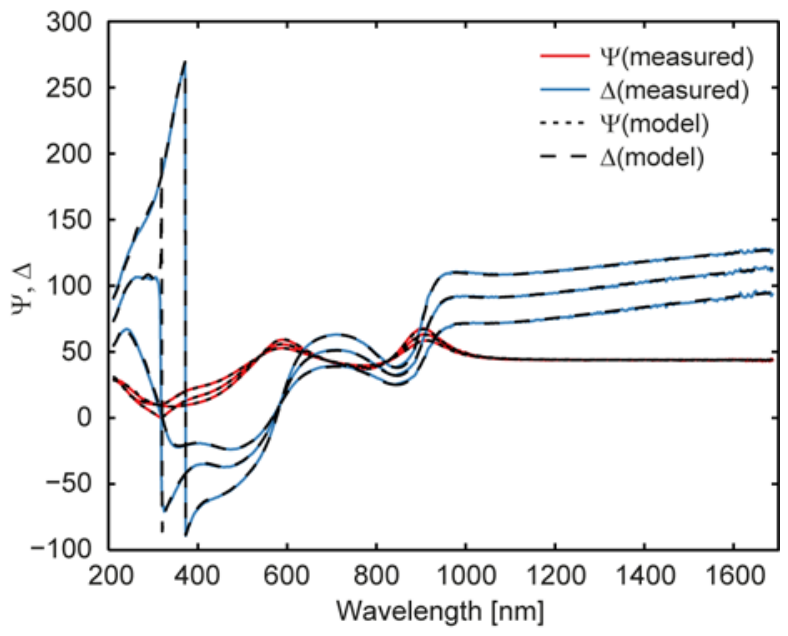

Figure S1. Representative spectroscopic ellipsometry data and stratified model fit for the sample with a SnS coated gold nanodot array on reflective support $\left(\mathrm{SiO}_{2} / \mathrm{Al}\right)$, fitted with an effective thickness $d_{\text {eff }}=24.9 \mathrm{~nm}$ and optical constants shown in Fig. $3 \mathrm{~b}$ of the main text.

\section{Optical properties of gold nanodots and their effect on the sample absorptance}

As stated in the main text, the numerical finite element method calculations were performed using the previously published properties of the gold nanodot arrays. ${ }^{S 1}$ Data for the bulk properties of gold were taken from Weaver and Frederikse. ${ }^{\mathrm{S2}}$ In addition, a correction for surface scattering was used, implemented via the permittivity according to Eq 1.4 of Coronado and Schatz, ${ }^{\mathrm{S3}}$ with the prefactor $(" A ")$ taken to unity and the effective inelastic mean free path $l_{\text {eff }}=4 \mathrm{~V} / \mathrm{S}=10.3 \mathrm{~nm}$ with $S$ being the average nanodot surface area and $V$ their volume. For the surface scattering correction the properties of gold were further represented by an electron density of $5.9 \times 10^{22} \mathrm{~cm}^{-3},{ }^{, 4}$ an electron effective mass of 1.0, ${ }^{55}$ a Fermi velocity of $1.4 \times 10^{6} \mathrm{~m} / \mathrm{s},{ }^{54}$ and a bulk inelastic mean free path of 33 $\mathrm{nm}$ based on its dc conductivity. The resulting damping with and without the surface scattering term is shown in Fig. S2a. One could also consider the addition of Landau damping ${ }^{56}$ and chemical interface damping ${ }^{57}$ to refine this estimate further. On the other hand, the spread in different data sets reported for bulk gold is already quite large in the visible to near infrared range. This has been shown to be caused by actual variations in the gold samples characterized, such as in grain sizes and the presence of voids, with higher quality samples (larger grains and fewer voids) displaying a lower damping for photon energies in the region dominated by free electron behavior below the gold interband transitions around $2.5 \mathrm{eV} .^{58}$ For comparison, we have also included the data from Johnson and Christy. ${ }^{59}$ The latter are similar to the data compiled by Lynch and Hunter in this range, ${ }^{510}$ and the damping part of the permittivity is considerably higher than those of Weaver and Frederikse. As shown in Fig. S2b, however, these variations have a relatively minor impact on the calculated absorptance in the gold nanodot array samples investigated in the present work. Even the branching ratio for absorption in the $\mathrm{SnS}$ coating versus in the $\mathrm{Au}$ is only slightly modified; the estimate for the integrated air mass 1.5 global photocurrent is reduced from 29.0 to $28.4 \mathrm{~mA} / \mathrm{cm}^{2}$ with the Johnson and Christy data. 

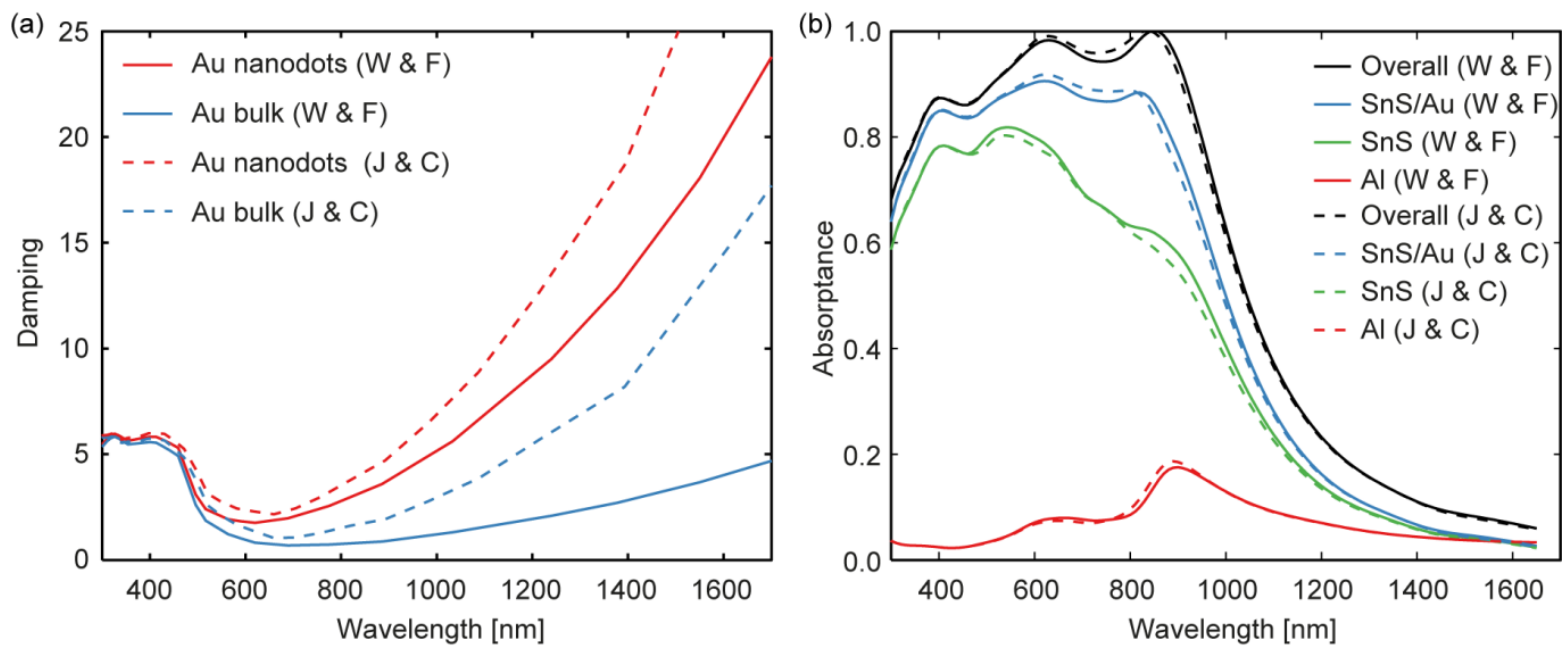

Figure S2. (a), Damping of bulk gold as reported by Weaver and Frederikse, ${ }^{\mathrm{S} 2}$ and the result after correction for surface scattering in the small nanodots. For comparison, the older data set of Johnson and Christy ${ }^{59}$ is also included with and without the surface scattering contribution. (b) Sample absorptance calculated using the two sets of data including surface scattering shown.

\section{Effect of aluminum interband transitions on overall absorptance}
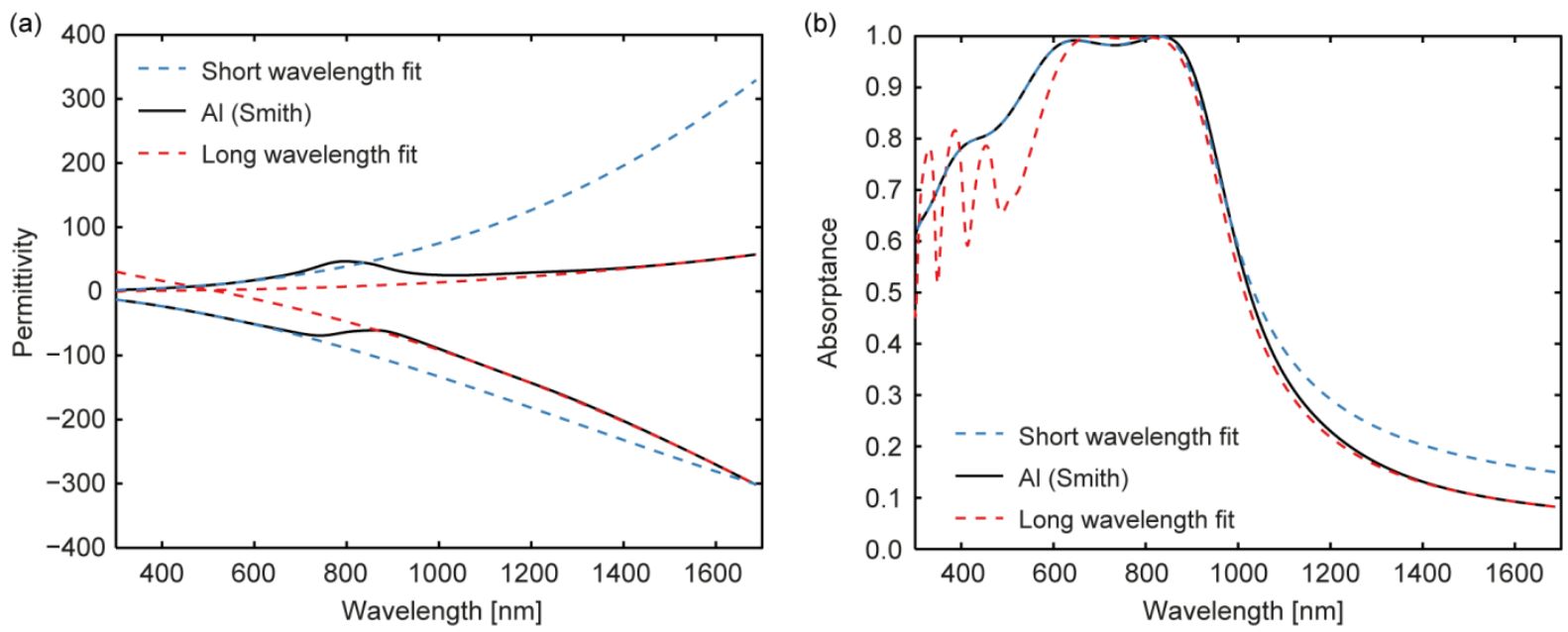

Figure S3. (a) Real (mostly negative) and imaginary (positive) components of the aluminum permittivity, as reported by Smith et al. ${ }^{511}$ For the purpose of excluding the effect of interband transitions in Al, Drude-type free electron behavior ${ }^{512}$ was imposed and fitted to the short- and long wavelength ranges, respectively. (b), Sample absorptance calculated using the aluminum data as well as the two Drude-fits. The main absorption feature is split into dual bands in all three cases. 


\section{Effect of coating filling factor on overall and individual layer absorptance}

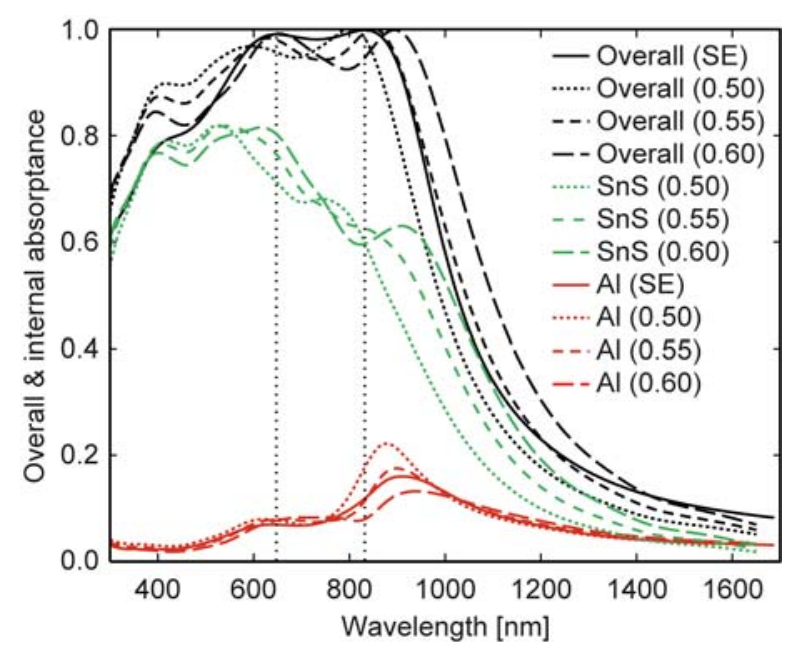

Figure S4. Overall absorptance and absorptance in the SnS and Al from SE and FEM calculations. The calculated data are for filling factors of $0.5,0.55$ and 0.6 , respectively, for the SnS/air mixture used to approximate the $10 \mathrm{~nm}$ conformal SnS coating of the array. The 0.55 filling factor clearly produces the best fit to the data. The corresponding photocurrents, based on the SnS absorptance and air mass 1.5 global irradiance data, are $27.1,29.0$ and $30.4 \mathrm{~mA} / \mathrm{cm}^{2}$, respectively. This demonstrates that this analysis is fairly robust with respect to the photocurrent estimate.

\section{Comparison of the absorption in SnS with and without gold nanodots}

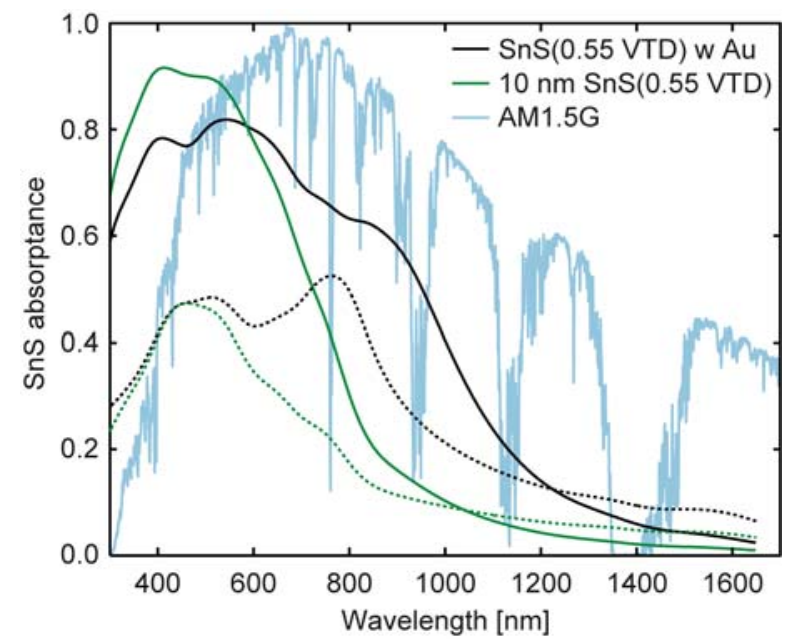

Figure S5. Calculated absorptance in $\mathrm{SnS}$ layers on top of a $55 \mathrm{~nm} \mathrm{SiO} \mathrm{S}_{2}$ spacer and Al reflector. The case of $10 \mathrm{~nm}$ SnS coating (modeled as a mixture of air and 55\% VTD SnS) on gold nanodots ( $w \mathrm{Au}$ ), is compared to a plain $10 \mathrm{~nm} \mathrm{SnS}$ film on the same reflective support. The dotted lines show the absorptance in the same type of nanocomposite and $\mathrm{SnS}$ layers when a very thick $\mathrm{SiO}_{2}$ spacer replaces the reflective Al layer. The normalized solar AM1.5G spectral photon flux is shown for reference. 


\section{Supporting references}

S1. Hägglund, C.; Zeltzer, G.; Ruiz, R.; Thomann, I.; Lee, H.-B.-R.; Brongersma, M. L.; Bent, S. F., Self-Assembly Based Plasmonic Arrays Tuned by Atomic Layer Deposition for Extreme Visible Light Absorption. Nano Lett. 2013, 13, 3352-3357.

S2. Weaver, J. H.; Frederikse, H. P. R., Optical Properties of Selected Elements. In Crc Handbook of Chemistry and Physics, 83 ed.; Lide, D. R., Ed. CRC Press: Boca Raton, FL, 2002; pp 133156.

S3. Coronado, E. A.; Schatz, G. C., Surface Plasmon Broadening for Arbitrary Shape Nanoparticles: A Geometrical Probability Approach. J. Chem. Phys. 2003, 119, 3926-3934.

S4. Berger, L. I., Optical Properties of Selected Inorganic and Organic Solids. In Crc Handbook of Chemistry and Physics, 83 ed.; Lide, D. R., Ed. CRC Press: 2002; pp 157-178.

S5. Thèye, M.-L., Investigation of the Optical Properties of Au by Means of Thin Semitransparent Films. Phys. Rev. B 1970, 2, 3060-3078.

S6. Hubenthal, F.; Hendrich, C.; Träger, F., Damping of the Localized Surface Plasmon Polariton Resonance of Gold Nanoparticles. Applied Physics B: Lasers \& Optics 2010, 100, 225-230.

S7. Persson, B. N. J., Polarizability of Small Spherical Metal Particles: Influence of the Matrix Environment. Surf. Sci. 1993, 281, 153-162.

S8. Aspnes, D. E.; Kinsbron, E.; Bacon, D. D., Optical Properties of Au: Sample Effects. Phys. Rev. B 1980, 21, 3290-3299.

S9. Johnson, P. B.; Christy, R. W., Optical-Constants of Noble-Metals. Phys. Rev. B 1972, 6, 43704379 .

S10. Lynch, D. W.; Hunter, W. R., Comments on the Optical Constants of Metals and an Introduction to the Data for Several Metals. In Handbook of Optical Constants of Solids, Palik, E. D., Ed. Academic Press: Burlington, 1997; Vol. I, pp 293-294.

S11. Smith, D. Y.; Shiles, E.; Inokuti, M., The Optical Properties of Metallic Aluminium. In Handbook of Optical Constants of Solids, Palik, E. D., Ed. Academic Press: Burlington, 1997; Vol. I, pp 397-400.

S12. Bohren, C. F.; Huffman, D. R., Absorption and Scattering of Light by Small Particles. WileyVCH: Weinheim, 1998; $p 544$. 